\title{
Lymphoma associated bone marrow necrosis with raised anticardiolipin antibody
}

\author{
P T Murphy, M Sivakumaran, M C Casey, A Liddicoat, J K Wood
}

\begin{abstract}
A case of high grade $B$ cell lymphoma presented with bone marrow necrosis, followed by development of extensive marrow fibrosis, the evolution of which was documented by serial magnetic resonance imaging and bone marrow trephine histology. A markedly raised anticardiolipin antibody titre at diagnosis suggests that lymphoma associated antiphospholipid syndrome may have contributed to the aetiology of the bone marrow necrosis. (f Clin Pathol 1998;51:407-409)
\end{abstract}

Keywords: lymphoma; bone marrow necrosis; anticardiolipin antibody

Bone marrow necrosis is a rare condition in which death of bone marrow stromal cells and haemopoietic cells occurs. Causes are multiple and include lymphoma. Necrotic marrow is eventually repopulated with haemopoietic cells but occasionally extensive fibrosis develops. We describe a case of bone marrow necrosis caused by high grade non Hodgkin's lymphoma in which recovery was associated with the development of extensive fibrosis. This evolution was documented not only histologically but also by serial magnetic resonance imaging (MRI) scans. In addition, anticardiolipin antibody titre was markedly raised at diagnosis, suggesting that lymphoma associated antiphospholipid syndrome may have contributed to the aetiology of the bone marrow necrosis.

\section{Case history}

Leicester Royal Infirmary, Leicester, UK

Department of Haematology P T Murphy

M Sivakumaran $\mathrm{J}$ K Wood

Department of Radiology

A Liddicoat

Department of Geriatric Medicine, Keele University, Stoke-on-Trent, UK M Casey

Correspondence to: Dr P T Murphy, Department of Haematology, Leicester Royal Infirmary, Leicester LE1 5WW, UK

Accepted for publication 20 January 1998
Serum lactic dehydrogenase $(\mathrm{LDH})$ was $2500 \mathrm{IU} /$ litre (normal range 350-700). Prothrombin time, activated partial thromboplastin time, thrombin time, and fibrin degradation products were all normal. Antinuclear factor, anti-double-stranded DNA, and serum immunoglobulins were within normal range.

Serology for Epstein-Barr virus, cytomegalovirus, and parvovirus were negative for active infection.

A radioisotope bone scan showed areas of increased uptake in spine, pelvis and femurs. Computerised tomography of chest and abdomen confirmed splenomegaly without lymphadenopathy. MRI of the thoracic and lumbar spines was performed: T1 weighted sagittal scans showed ring like and serpiginous areas of high signal surrounding focal areas of low signal. These changes were seen in all the visualised vertebral bodies. In addition, more diffuse areas of low signal were seen in the sixth, seventh, and eight thoracic vertebral bodies. Degenerative changes were also noted at multiple levels (fig 1A). On T2 weighted scans, the low signal central foci were surrounded by a larger peripheral zone of high signal intensity (fig 1B). The pattern of disease would have been compatible with marrow necrosis although the signal changes were atypical.

Within days of admission, the patient developed melaena. Gastroscopy showed an ulcer in the greater curve of the stomach, biopsy of which suggested adenocarcinoma. A week after admission, repeat bone marrow aspirate again showed abnormal necrotic cells but direct immunofluorescence staining of bone marrow aspirate cytospins for light chains revealed large lymphoid cells with a kappa:lambda light chain ratio of $>20: 1$, despite poor morphology. After six weeks, platelet count had recovered to over $100 \times 10^{9} /$ litre and neutrophil count to normal, with $\mathrm{LDH}$ reduced to $706 \mathrm{IU} /$ litre. Trephine biopsy continued to show focal areas of necrosis but also bone marrow regeneration with areas of myeloid and megakaryocytic hyperplasia. There were also small focal areas of abnormal lymphoid tissue, suggestive of lymphoma, while reticulin was now markedly and diffusely increased. At that time, the patient underwent a partial gastrectomy with complete removal of a moderately differentiated adenocarcinoma.

A further trephine biopsy, 10 weeks after initial presentation, showed resolution of necrosis but persistent focal lymphomatous infiltration with progressive diffuse increase in reticulin as well as an increase in osteoblasts and fibroblasts. The patient required readmission after a further four weeks with recurrence of pancytopenia ( $\mathrm{Hb} 5.7 \mathrm{~g} / \mathrm{dl}$, WBC $0.6 \times 10^{9} /$ litre, and platelet count $10 \times 10^{9} /$ litre) and development of widespread skin nodules. Skin biopsy showed immunoblastic B cell non-Hodgkin's 

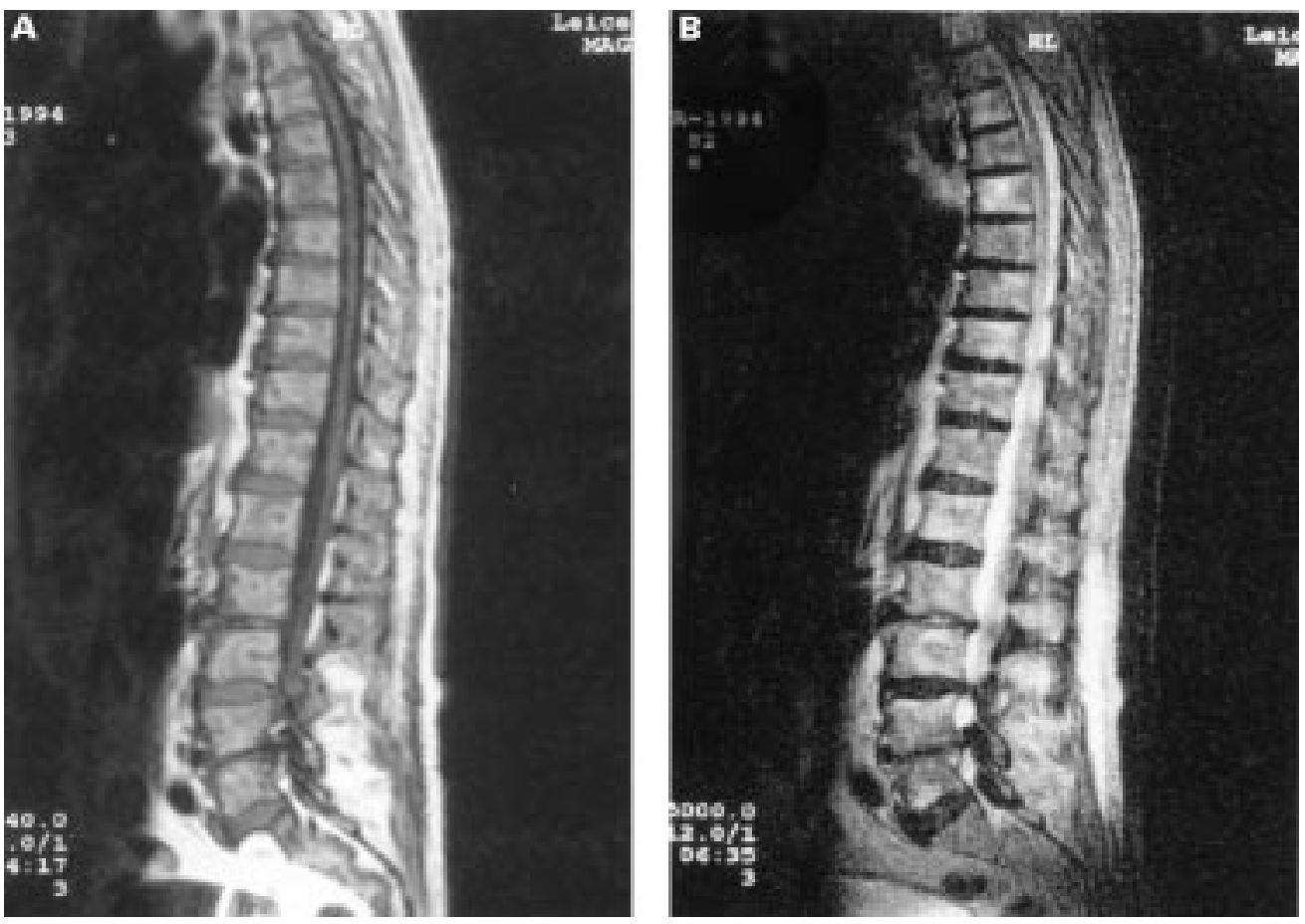

Figure 1 Magnetic resonance imaging of thoracic and lumbar spines at presentation. (A) T1 weighted sagittal scan showing focal areas of low signal with high signal periphery. (B) T2 weighted sagittal scan with focal areas of low signal, many associated with diffuse high signal change.

lymphoma with plasmacytoid features. On repeat MRI, T1 weighted sagittal scans showed diffuse loss of signal throughout the vertebrae and high signal around the L2/3 and L4/5 discs, secondary to degenerative disease and haemorrhage (fig 2A). On T2 weighted sagittal scans, there was a more inhomogeneous pattern with multiple rounded areas of low signal and ring like areas of high signal, some of

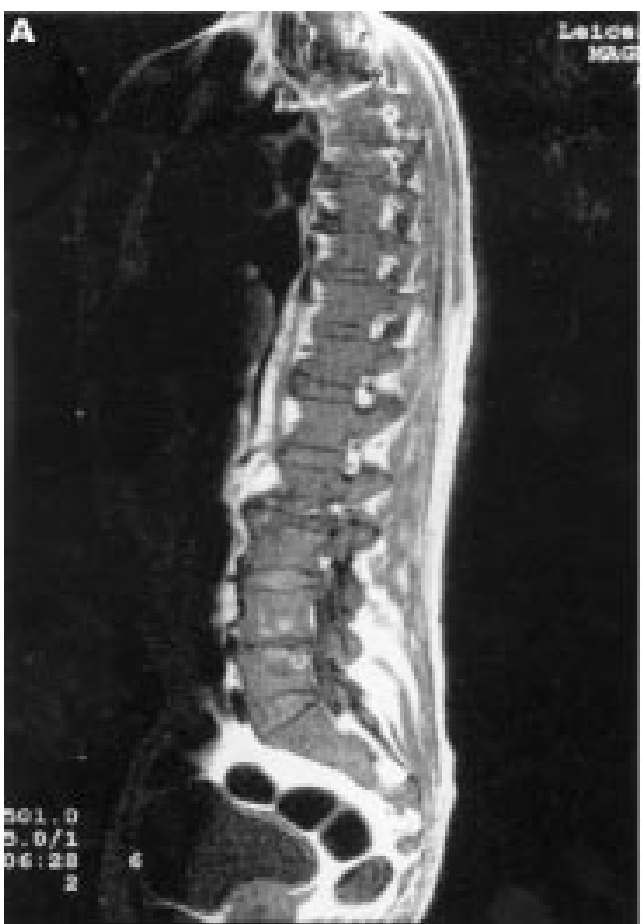

which had a low signal central focus (fig 2B). The diffuse loss of signal on the $\mathrm{T} 1$ weighted scans was consistent with diffuse malignant infiltration, marrow reconversion, or fibrosis, but $\mathrm{T} 2$ weighted scans suggested a more focal abnormality. The patient was started on CHOP chemotherapy with rapid reduction in skin nodules, but died four months after initial presentation from pneumonia. Permission for

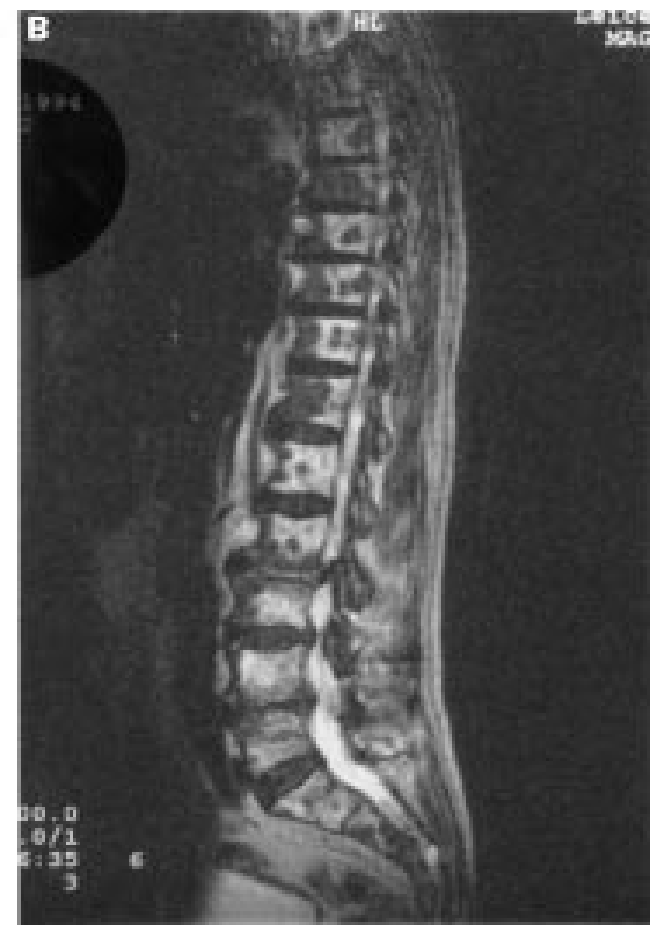

Figure 2 Repeat magnetic resonance imaging of thoracic and lumbar spines. (A) T1 weighted sagittal scan with diffuse loss of signal throughout the vertebral bodies and haemorrhagic change around degenerate discs. (B) T2 weighted sagittal scan showing multiple focal abnormalities of low intensity and high intensity ring-like areas. 
necropsy was refused. Subsequently, following two reports of bone marrow necrosis associated with antiphospholipid syndrome, ${ }^{23}$ an IgG phospholipid (GPL) anticardiolipin antibody test on stored serum taken from our patient at diagnosis was positive at $>100 \mathrm{U} /$ litre.

\section{Discussion}

Bone marrow necrosis associated with high grade B cell lymphoproliferative disease is rare, with only nine previously reported cases diagnosed during life. ${ }^{4}$ We are aware of only one previous report describing the use of MRI at diagnosis in three cases of lymphoma associated with bone marrow necrosis. ${ }^{5}$ In that report, MRI showed two distinct patterns of necrosis. The first pattern (multifocal abnormalities with low intensity on $\mathrm{T} 1$ weighted images and very high intensity on T2 weighted images) in two patients with non-Hodgkin's lymphoma was consistent with fluid filled central necrotic regions, while the second pattern (extensive ring-like lesions) in a patient with Hodgkin's disease was more compatible with avascular necrosis of bone. The MRI findings in our patient at presentation of bone marrow necrosis showed extensive ring like lesions but were dissimilar to the second pattern, as high signal was present on T2 weighted scans. However, in view of the histological findings of extensive bone marrow necrosis with only slight lymphomatous infiltration, these MRI findings appear to be another unique pattern of bone marrow necrosis.

Our case is also unusual because of evolution of bone marrow necrosis into extensive marrow fibrosis. Interpretation of repeat MRI three months after initial presentation is complicated by progression of focal lymphomatous marrow infiltration in addition to the development of extensive fibrosis. The abnormal patterns on the T1 and T2 weighted images are not entirely typical of either lymphomatous involvement or marrow fibrosis but, because trephine biopsy at the time showed extensive and diffuse marrow fibrosis with only focal areas of lymphoma, the MRI findings might largely be explained by the marrow fibrosis.

After the death of our patient, two reports were published suggesting that primary antiphospholipid syndrome may have had a central role in the pathogenesis of bone marrow necrosis in two young women. ${ }^{23}$ We subsequently found a markedly raised anticardiolipin antibody titre in our patient's serum, stored at diagnosis. It is therefore intriguing to speculate that lymphoma associated antiphospholipid syndrome may have played a role in the aetiology of the bone marrow necrosis at diagnosis. In view of our case and the previous reports of bone marrow necrosis associated with primary antiphospholipid syndrome, we suggest that investigations for antiphospholipid syndrome should be carried out in all subsequent cases of bone marrow necrosis. Although we excluded active infection by Ebstein-Barr virus, cytomegalovirus, and parvovirus, there is also the possibility that some other infection may have been the initial event leading to the bone marrow necrosis at presentation.

1 Froberg PK, Braunstein EM, Buckwalter KA. Osteonecrosis, transient osteoporosis and transient bone marrow edema: current concepts. Radiol Clin North $\mathrm{Am}$ 1996;34:273-91.

2 Bulvik S, Aronson I, Ress S, Jacobs P. Extensive bone marrow necrosis associated with antiphospholipid antibodies. Am f Med 1995;98:572-4.

3 Paydas S, Kocak R, Zorludemir S, Baslamisli F. Bone marrow necrosis in antiphospholipid syndrome. $\mathcal{f}$ Clin Pathol 1997;50:261-2.

4 Ranaghan L, Morris TCM, Desai ZR, Markey GM. Bone Ranaghan L, Morris TCM, Desai ZR, Markey
marrow necrosis. Am $\mathcal{J}$ Med 1994;47:225-8.

5 Weissman DE, Negandank WG, Al-Katib AM, Smith MR. Bone marrow necrosis in lymphoma studied by magnetic resonance imaging. Am F Hematol 1992;40:42-6. 